\title{
An introduction to tight closure
}

KAREN E. SMITH Department of Mathemathics, University of Michigan, Ann Arbor, MI, 48109, kesmith@math.lsa.umich.edu

\section{INTRODUCTION}

This is an expanded version of my lecture at the Conference in Commutative Algebra and Algebraic Geometry in Messina Italy in June 1999. The purpose of the talk was to give a brief introduction to the subject of tight closure, aimed at commutative algebraists who have not before studied this topic. The first part focused mainly on the definition and basic properties, with the second part focusing on some applications to algebraic geometry, particularly to global generation of adjoint linear series. These lecture notes follow even more closely a series of two lectures I gave in Kashikojima, Japan, at the Twentieth Annual Japanese Conference in Commutative Algebra the previous fall, and were distributed also in conjunction with that conference. I wish to thank the organizers of both conferences, Professors Restuccia and Herzog for the European conference, and Professors Hashimoto and Yoshida, for the Japanese conference. Both events were a smashing success. Special thanks are due also to Rosanna Utano, for help editing the tex file.

Tight closure was introduced by Mel Hochster and Craig Huneke in 1986 [18]. Today it is still a subject of very active research, with an ever increasing list of applications. Applications include areas like the study of Cohen-Macaulayness. For example, the famous Hochster-Roberts theorem on the Cohen-Macaulayness of rings of invariants has a simple tight closure proof $[18]$. Also, the existence of big Cohen-Macaulay algebras for rings containing a field was proved with ideas from tight closure [20], and the existence of "arithmetic Macaulayfications" in some cases was discovered with tight closure [3], 29]. Tight closure has provided us with greater insight into integral closure, and into the homological theorems that grew out of Serre's work on multiplicities. For example, it gives us simple proofs of the Briançon-Skoda Theorem, the Syzygy Theorem of Evans and Griffith and of the monomial conjecture (in equi-characteristic) [18. Tight closure provided the inspiration for results on the simplicity of rings of differential operators on certain 
rings of invariants 45, and it has produced "uniform" Artin-Rees theorems 22]. There are also numerous applications to and connections with algebraic geometry, such as in the study of singularities [46], [38], [11], of vanishing theorems [12], [25], [14], and of adjoint linear series [41], 44]. In Section 3, I will summarize some of these applications to algebraic geometry, although of course, there will not be enough time to do any of them any justice.

Let us begin with our first task: to introduce the definition of tight closure before tackling its main properties in the next section.

Tight closure is a closure operation performed on ideals in a commutative, Noetherian ring containing a field (that is, of "equi-characteristic"). The tight closure of an ideal $I$ is an ideal $I^{*}$ containing $I$. The definition is based on reduction to characteristic $p$, where the Frobenius (or $p-t h$ power map) is then used. To keep things as simple as possible, we treat only the characteristic $p$ case here.

DEFINITION 1.1 Let $R$ be a Noetherian domain of prime characteristic $p$, and let $I$ be an ideal with generators $\left(y_{1}, \ldots, y_{r}\right)$. An element $z$ is defined to be in the tight closure $I^{*}$ if there exists a non-zero element $c$ of $R$ such that

$$
c z^{p^{e}} \in\left(y_{1}^{p^{e}}, \ldots, y_{r}^{p^{e}}\right)
$$

for all $e \gg 0$.

Loosely speaking, the tight closure consists of all elements that are "almost" in $I$ as far as the Frobenius map is concerned. Indeed, if we take the $p^{e}-t h$ root of $(*)$ above, we see that

$$
c^{1 / p^{e}} z \in I R^{1 / p^{e}}
$$

for $e \gg 0$. As $e$ goes to infinity, $1 / p^{e}$ goes to zero, so in some sense $c^{1 / p^{e}}$ goes to 1 (this idea can be made precise with valuations). So $z$ is "almost in" $I$, at least after applying the Frobenius map.

It is not important to restrict to the case where $R$ is a domain; we can define tight closure in an arbitrary Noetherian ring of characteristic $p$ by requiring that $c$ is not in any minimal prime. However, because most theorems about tight closure reduce to the domain case, we treat only the domain case in this lecture.

EXAMPLE 1.2 Example. Let $R$ be the hypersurface ring

$$
\frac{k[x, y, z]}{\left(x^{3}+y^{3}-z^{3}\right)},
$$

where $k$ is any field whose characteristic is not 3. Then

$$
(x, y)^{*}=\left(x, y, z^{2}\right) .
$$

Indeed, if $k$ has characteristic $p$, we can write

$$
\left(z^{2}\right)^{q}=\left(x^{3}+y^{3}\right)^{\frac{2 q-r}{3}} z^{r}
$$


where $r=1$ or 2 and $q=p^{e}$. Expanding this expression as

$$
z^{r} \sum\left(\begin{array}{c}
\frac{2 q-r}{3} \\
i
\end{array}\right) x^{3 i} y^{2 q-r-3 i},
$$

it is easy to see that each monomial $x^{m} y^{n}$ appearing in the sum has either $m \geq q$ or $n \geq q$ unless both $m$ and $n$ equal $q-1$ (which only happens in the case where $q=1 \bmod 3)$. So we can take $c=x$ (or $y)$, and conclude that $c\left(z^{2}\right)^{q} \in\left(x^{q}, y^{q}\right)$ for all $q=p^{e}$. Thus $z^{2} \in(x, y)^{*}$. A similar argument can be used to show that $z$ is not in $(x, y)^{*}$. Because this works for all $p$ (except $\left.p=3\right)$, we declare that $z^{2}$, but not $z$, is in the tight closure of $(x, y)$ also in characteristic zero. So $(x, y)^{*}=\left(x, y, z^{2}\right)$ in every characteristic $p \geq 0$ except $p=3$.

\section{MAIN PROPERTIES OF TIGHT CLOSURE}

The definition of tight closure takes some getting used to. Fortunately, one can understand many applications of tight closure if one simply accepts the following properties of tight closure as axioms:

\section{Main Properties of Tight Closure}

(1) If $R$ is regular, then all ideals of $R$ are tightly closed.

(2) If $R \hookrightarrow S$ is an integral extension, then $I S \cap R \subset I^{*}$ for all ideals $I$ of $R$.

(3) If $R$ is local, with system of parameters $x_{1}, \ldots, x_{d}$, then $\left(x_{1}, \ldots, x_{i}\right): x_{i+1} \subset$ $\left(x_{1}, \ldots, x_{i}\right)^{*}$ ("Colon Capturing").

(4) If $\mu$ denotes the minimal number of generators of $I$, then $\overline{I^{\mu}} \subset I^{*} \subset \bar{I}$, where for any ideal $J, \bar{J}$ denotes the integral closure of $J$.

(5) If $R \rightarrow S$ is any ring map, $I^{*} S \subset(I S)^{*}$ ("Persistence").

For the remainder of this section, we will discuss these five main properties, their proofs and main consequences. Some of the five require some mild hypotheses; precise statements will be given. All of them are true in any equicharacteristic ring (although Property 2 is not interesting in characteristic zero). All of them are quite elementary to prove, at least in the main settings, with the exception of Property 5 which requires a new idea. We will stick to the prime characteristic case, and simply remark that "by reduction to characteristic $p$ ", one can prove the characteristic zero case without essential difficulty.

Note that one important property is omitted from the list. Any decent closure operation ought to commute with localization, but amazingly, we still do not know that tight closure does.

Open Problem If $U$ is a multiplicative system in a ring $R$, is

$$
I^{*} R\left[U^{-1}\right]=\left(I R\left[U^{-1}\right]\right)^{*} ?
$$


It is easy to see that one direction holds, namely, $I^{*} R\left[U^{-1}\right] \subset\left(\operatorname{IR}\left[U^{-1}\right]\right)^{*}$. Indeed, if $z \in I^{*}$, then we have the equations $c z^{p^{e}}=a_{1 e} y_{1}^{p^{e}}+\cdots+a_{r e} y_{r}^{p^{e}}$ in $R$. Expanding to $R\left[U^{-1}\right]$, the same equations show that $\frac{z}{1}$ is in the tight closure of $\operatorname{IR}\left[U^{-1}\right]$. This is a very special case of Property 5 above. On the other hand, the other direction is not known in any non-trivial case (see, however, [2], 43]).

The localization problem is probably the biggest open problem in tight closure theory. It is remarkable that the theory is so powerful while such a basic question remains unsolved. The power is derived from the five main properties above, which we now discuss.

\section{Property One: All ideals are tightly closed in a regular ring.}

It is easy to see why all ideals are tightly closed in a regular ring. For example, consider the special case where $(R, m)$ is local domain and the Frobenius map is finite. This is not a very restrictive assumption from our point of view, because we are usually interested in the local case anyway; also the Frobenius map is finite in a large class of interesting rings - for example, for any algebra essentially of finite type over a perfect field or for any complete local ring with a perfect residue field.

We have a descending chain of subrings of $R$

$$
R \supset R^{p} \supset R^{p^{2}} \supset R^{p^{3}} \supset \ldots
$$

Because $R$ is regular, the ring $R$ is a free module considered over each one of the subrings $R^{p^{e}}$. Indeed, the Frobenius map is flat for any regular ring, but because we have assumed that $R$ is local and the Frobenius map is finite, we actually get that $R$ is free over $R^{p^{e}}$. This means that, for any non-zero $c$, we can find an $R^{p^{e}}$-linear splitting

$$
\begin{gathered}
R \stackrel{\phi}{\rightarrow} R^{p^{e}} \\
c \mapsto 1
\end{gathered}
$$

so long as $e$ is large enough that $c$ is not in the expansion of the maximal ideal of $R^{p^{e}}$ to $R$ (that is, $c \notin m^{\left[p^{e}\right]}$, where $m^{\left[p^{e}\right]}$ denotes the ideal of $R$ generated by the $p^{e}-t h$ powers of the generators of $m$ ).

Now if we have an ideal $I=\left(y_{1}, \ldots, y_{r}\right)$ of $R$ and an element $z \in I^{*}$, then we can find equations

$$
c z^{p^{e}}=a_{1} y_{1}^{p^{e}}+\cdots+a_{r} y_{r}^{p^{e}}
$$

for all large $e$. Applying the $R^{p^{e}}$-linear map $\phi$ above, we see that

$$
z^{p^{e}}=\phi\left(a_{1}\right) y^{p^{e}}+\cdots+\phi\left(a_{r}\right) y_{r}^{p^{e}}
$$

where each coefficient $\phi\left(a_{i}\right)$ is some element of $R^{p^{e}}$. By taking the $p^{e}-t h$ root of this equation, we see that $z$ is an $R$-linear combination of $y_{1}, \ldots, y_{r}$. Thus $z \in I$, and $I^{*}=I$ for all ideals of $R$.

This completes the proof that all ideals are tightly closed in a regular ring, at least in the special case we considered. The general case (of prime characteristic) is not much harder. The point is that flatness of Frobenius in a regular ring. See 18].

Property Two: Elements mapped to $I$ after integral extension are in $I^{*}$. 
We now prove Property 2: if $R \hookrightarrow S$ is an integral extension of domains of prime characteristic, and $I$ is an ideal of $R$, then $I S \cap R \subset I^{*}$.

The following lemma will be useful also in the proof of Property 3.

Key Lemma If $R \hookrightarrow S$ is a module finite extension of domains, and $d$ is any fixed non-zero element of $S$, then there is an $R$-linear map, $S \stackrel{\phi}{\rightarrow} R$ sending $d$ to a non-zero element of $R$.

The point in the proof of the Lemma is that after tensoring with the fraction field, $K$, of $R$, we have an inclusion $K \hookrightarrow K \otimes_{R} S$, where the latter is simply a finite dimensional vector space over $K$. So of course there is a $K$-linear splitting $K \otimes S \stackrel{\psi}{\rightarrow} K$ sending $1 \otimes d$ to 1 . Thinking of $S$ as a subset of $K \otimes S$, we look at where $\psi$ sends each of a set of $R$-module generators $\left\{s_{1}, \ldots, s_{d}\right\}$ for $S$, say $\psi\left(s_{i}\right)=\frac{r_{i}}{t_{i}} \in K$. Now we can define $\phi$ to be the map $t \psi$ where $t$ is the product of the $t_{i}$. This map is $R$-linear, and sends each $s_{i}$ to an element of $R$. The lemma is proved.

To prove Property 2 , let $z \in R$ be any element in $I S \cap R$. This means we can write

$$
z=a_{1} y_{1}+\cdots+a_{r} y_{r}
$$

where $a_{i} \in S$ and the $y_{i}$ 's generate $I$. Because this expression involves only finitely many elements from $S$ there is no loss of generality in assuming $S$ is module finite over $R$. Now, raising this equation to the $p^{e}-t h$ power, we have

$$
z^{p^{e}}=a_{1}^{p^{e}} y_{1}^{p^{e}}+\cdots+a_{r}^{p^{e}} y_{r}^{p^{e}} .
$$

Using the lemma, we find an $R$-linear map $S \stackrel{\phi}{\rightarrow} R$ sending 1 to some non-zero element $c \in R$. Applying $\phi$ to this equation, we have

$$
c z^{p^{e}}=\phi\left(a_{1}^{p^{e}}\right) y_{1}^{p^{e}}+\cdots+\phi\left(a_{r}^{p^{e}}\right) y_{r}^{p^{e}} .
$$

This is an equation now in $R$, showing that $z \in I^{*}$. Property 2 is proved.

Essentially the same argument shows the stronger property: if $R \hookrightarrow S$ is an integral extension of prime characteristic domains and $I$ is an ideal of $R$, then $(I S)^{*} \cap R \subset I^{*}$.

Property 2 , unlike the other four properties, is interesting only in prime characteristic. For example, if $R$ is any normal domain containing $\mathbb{Q}$, then $R$ splits off of every finite integral extension $S$ (using the trace map). In this case, $I S \cap R=I$ for every ideal of $R$ and every integral extension $S$.

Property 2 can be phrased in terms of the absolute integral closure. For any domain $R$, the absolute integral closure $R^{+}$of $R$ is the integral closure of $R$ in an algebraic closure of its fraction field. In other words, $R^{+}$is the direct limit of all finite integral extensions of $R$. Property 2 can be stated: $I R^{+} \cap R \subset I^{*}$ for all ideals $I$ of $R$. This leads to the following interesting problem.

Open Problem Let $R$ be a domain of prime characteristic. Is $I R^{+} \cap R=I^{*}$ for all ideals $R$ ?

In addition to providing a very nice characterization of tight closure, an affirmative answer to this question would immediately solve the localization problem. 
Indeed, it is easy to check that the closure operation defined by expansion to the absolute integral closure and contraction back to $R$ commutes with localization.

There is no non-trivial class of rings in which this open problem has been solved. However, we do have the following result.

THEOREM 2.1 38 Let $R$ be a locally excellen 7 domain of prime characteristic. Then $I^{*}=I R^{+} \cap R$ for all parameter ideals $I$ of $R$.

A "parameter ideal" is any ideal $I$ generated by $n$-elements where $n$ is the height of $I$; if $R$ is local, an ideal is a parameter ideal if and only if it is generated by part of a system of parameters.

As we see from the theorem, tight closure commutes with localization for parameter ideals. However, this does not follow from the theorem because this fact is used in its proof. See instead [2].

The proof of this theorem is somewhat involved, so we do not sketch it here; see [38. The result has been generalized to a larger class of ideals, including ideals generated monomials in the parameters, by Aberbach [1].

\section{Property Three: Colon Capturing.}

Property 3, the colon capturing property of tight closure, is particularly instrumental in applications of tight closure to problems about Cohen-Macaulayness. Of course, if $R$ is a Cohen-Macaulay local ring with system of parameters $x_{1}, \ldots, x_{d}$, then by definition,

$$
\left(x_{1}, \ldots, x_{i}\right): x_{i+1} \subset\left(x_{1}, \ldots, x_{i}\right)
$$

for each $i=1,2, \ldots, d-1$. Colon capturing says that, even for rings that are not Cohen-Macaulay, the colon ideal $\left(x_{1}, \ldots, x_{i}\right): x_{i+1}$ is at least contained in $\left(x_{1}, \ldots, x_{i}\right)^{*}$. Loosely speaking, tight closure captures the failure of a ring to be Cohen-Macaulay.

We now prove the colon capturing property of tight closure: if $R$ is a local domain (satisfying some mild hypothesis to be made soon precise) and $x_{1}, \ldots, x_{d}$ is a system of parameters for $R$, then

$$
\left(x_{1}, \ldots, x_{i}\right): x_{i+1} \subset\left(x_{1}, \ldots, x_{i}\right)^{*}
$$

for each $i=1, \ldots, d-1$.

Let us first assume that $R$ is complete. In this case, we can express $R$ as a module finite extension of the power series subring $k\left[\left[x_{1}, \ldots, x_{d}\right]\right]$, where $k$ is a field isomorphic to the residue field of $R$.

Suppose that $z \in\left(x_{1}, \ldots, x_{i}\right):_{R} x_{i+1}$. Consider the ring $A$ contained in $R$ obtained by adjoining the element $z$ to the power series ring $k\left[\left[x_{1}, \ldots, x_{d}\right]\right]$. Observe that the ring $A$ is Cohen-Macaulay; in fact, $A$ is a hypersurface ring because its dimension is $d$ and its embedding dimension is $d+1$ (or $d$, if $z$ happens to be in power series ring already).

Now, because $z \in\left(x_{1}, \ldots, x_{i}\right):_{R} x_{i+1}$, we can write

$$
z x_{i+1}=a_{1} x_{1}+\cdots+a_{i} x_{i}
$$

\footnotetext{
${ }^{1}$ Virtually all rings the commutative algebraist on the street is likely to run across are locally excellent, but see [34] for a definition.
} 
for some elements $a_{i}$ in $R$. Raising this equation to the $p^{e}-t h$ power, we have

$$
z^{p^{e}} x_{i+1}^{p^{e}}=a_{1}^{p^{e}} x_{1}^{p^{e}}+\cdots+a_{i}^{p^{e}} x_{i}^{p^{e}}
$$

Because the inclusion $A \hookrightarrow R$ is a module finite extension, we can use the Key Lemma to find an $A$-linear map $R \stackrel{\phi}{\rightarrow} A$ sending 1 to some non-zero element $c \in A$. This yields equations

$$
c z^{p^{e}} x_{i+1}^{p^{e}}=\phi\left(a_{1}^{p^{e}}\right) x_{1}^{p^{e}}+\cdots+\phi\left(a_{i}^{p^{e}}\right) x_{i}^{p^{e}}
$$

where the $\phi\left(a_{j}^{p^{e}}\right)$ are just some elements of $A$.

In other words,

$$
c z^{p^{e}} \in\left(x_{1}^{p^{e}}, \ldots, x_{i}^{p^{e}}\right):_{A} x_{i+1}^{p^{e}}
$$

in the ring $A$. But $A$ is Cohen-Macaulay, and $x_{1}^{p^{e}}, \ldots, x_{d}^{p^{e}}$ is a system of parameters for $A$, so we see

$$
c z^{p^{e}} \in\left(x_{1}^{p^{e}}, \ldots, x_{i}^{p^{e}}\right)
$$

for all $e$. This shows that $z \in\left(x_{1}, \ldots, x_{i}\right)^{*}$ in $R$ (also in $A$, but it is $R$ we care about). Thus $\left(x_{1}, \ldots, x_{i}\right):_{R} x_{i+1} \subset\left(x_{1}, \ldots, x_{i}\right)^{*}$, and the proof of the colon capturing property is complete - at least for complete local domains.

Inspecting the proof, we see that we have not used the completeness of $R$ in a crucial way: what we need is that $R$ the domain is a finite extension of a regular subring. So this proof also works for algebras essentially of finite type over a field (the required regular subring is supplied by Noether normalization) and in many other settings. In fact, colon capturing holds for any ring module finite and torsion free over a regular ring. See [18] and [23] for different proofs and more general statements.

The philosophy of colon capturing holds for other ideals involving parameters. For example, if $I$ and $J$ are any ideals generated by monomials in a system of parameters $\left\{x_{0}, \ldots, x_{d}\right\}$, one can compute $I: J$ formally as if the $x_{i}$ 's are the indeterminates of a polynomial ring. Then the actual colon $I: J$ is contained in the tight closure of the 'formal' colon ideal. Furthermore, even more is true: we have $I^{*}: J$ is contained in the tight closure of the formal colon ideal. Essentially the same proof gives these stronger results with very small effort. For an explicit example, let $x_{0}, \ldots, x_{d}$ be a system of parameters in a domain $R$. Then

$$
\left(x_{0}^{t}, \ldots, x_{d}^{t}\right):\left(x_{0} x_{1} \ldots x_{d}\right) \subset\left(x_{0}^{t-1}, \ldots, x_{d}^{t-1}\right)^{*}
$$

and even

$$
\left(x_{0}^{t}, \ldots, x_{d}^{t}\right)^{*}:\left(x_{0} x_{1} \ldots x_{d}\right) \subset\left(x_{0}^{t-1}, \ldots, x_{d}^{t-1}\right)^{*} .
$$

One reason for tight closure's effectiveness is that these sorts of manipulations can often help us prove a general statement about parameters if we already have an argument for a regular sequence.

\section{Some Consequences of the First Three Properties.}

It follows immediately from the colon capturing property that if $R$ is a local ring in which all ideals are tightly closed, then $R$ must be Cohen-Macaulay. Indeed, 
if all parameter ideals are tightly closed, then colon capturing implies that $R$ is Cohen-Macaulay. This leads us to define two important new classes of rings.

DEFINITION 2.2 A ring $R$ is weakly F-regular if all ideals are tightly closed. A ring $R$ is F-rational if all parameter ideals are tightly closed.

So far we have seen the following implications: Regular $\Longrightarrow$ weakly F-regular $\Longrightarrow$ F-rational $\Longrightarrow$ Cohen-Macaulay. The first implication is Property 1, while the last implication is Property 3.

The reason the adjective "weakly" modifies "F-regular" above goes back to the localization problem. Unfortunately, we do not know whether the property that all ideals are tightly closed is preserved under localization. The term "F-regular" is reserved for rings $R$ in which all ideals are tightly closed not just in $R$, but also in every localization of $R$. That is, we have the following special case of the localization problem:

Open Problem If $R$ is weakly $F-$ regular, and $U \subset R$ is any multiplicative system, is the localization $R\left[U^{-1}\right]$ also weakly $F$-regular?

This problem is much easier than the localization problem itself. Indeed, it has been shown in a number of cases. For example, Hochster and Huneke showed the answer is yes when $R$ is Gorenstein [19], [21. This was later generalized to the $\mathbb{Q}-$ Gorenstein case, and even to the case where there are only isolated non $\mathbb{Q}$-Gorenstein points, by MacCrimmon [33]. Using this, it is possible to see that weakly F-regular is equivalent to F-regular in dimensions three and less. (These statements require some mild assumption on $R$, such as excellence). Recently, an affirmative answer was given also for finitely generated $\mathbb{N}$-graded algebras over a field [32]. By contrast, the full localization problem has not been solved in any of these cases.

The problem is reminiscent of an analogous problem in commutative algebra that looked quite difficult in the mid-century: is the localization of a regular ring still regular? With Serre's introduction of homological algebra to commutative algebra, the problem suddenly became quite easy. Perhaps a similar revelation is necessary in tight closure theory.

Returning to the applications of the first three properties, we now prove the following easy, but important, theorem.

THEOREM 2.3 18 Let $R \subset S$ be an inclusion of rings that splits as a map of $R$-modules. If $S$ is (weakly) F-regular, then $R$ is (weakly) F-regular.

The proof is simple. Suppose that $I$ is an ideal of $R$ and that $z \in I^{*}$. This means that for some non-zero $c, c z^{p^{e}} \in I^{\left[p^{e}\right]}$ where $I^{\left[p^{e}\right]}$ denotes the ideal generated by the $p^{e}-t h$ powers of the generators of $I$. Expanding to $S$, we have $c z^{p^{e}} \in(I S)^{\left[p^{e}\right]}$, so that $z \in(I S)^{*}$. But all ideals of $S$ are tightly closed, and so $z \in I S$. Now applying the splitting $S \rightarrow R$ (which sends 1 to $1 R$-linearly), we see that $z \in I$ in $R$ as well. This completes the proof.

The importance of this Theorem lies in the following corollaries. 
COROLLARY 2.4 Any ring (containing a field) which is a direct summand of a regular ring is Cohen-Macaulay.

The proof is obvious: a regular ring is F-regular by Property 1, so any direct summand is also F-regular. By Property 3, this summand is Cohen-Macaulay.

COROLLARY 2.5 (The Hochster-Roberts Theorem) The ring of invariants of a linearly reductive group acting linearly on a regular ring is Cohen-Macaulay.

This is essentially a special case of the previous corollary because the so-called Reynold's operator gives us a splitting of $R^{G}$ from $R$.

We emphasize that both the Theorem and its corollaries make sense and are true in characteristic zero. Thus even though there are very fewer linearly reductive groups in prime characteristic, the Hochster-Roberts Theorem for reductive groups over the complex numbers has been proved here by reduction to characteristic $p$. To be fair, we have not proved Properties 1 and 3 in characteristic zero (nor even given a precise definition of tight closure in characteristic zero). However, if one accepts the existence of a closure operation in characteristic zero satisfying Properties 1 and 3 , then we have proved that the Hochster-Roberts Theorem follows.

We now mention one of the crown jewels of tight closure theory.

THEOREM 2.6 20] Let $R$ be an excellent local domain of prime characteristic. Then the absolute integral closure $R^{+}$of $R$ is a Cohen-Macaulay $R$-module.

We can see that this must be true as follows. Let $x_{1}, \ldots, x_{d}$ be a system of parameters. Suppose $z \in\left(x_{1}, \ldots, x_{i}\right): x_{i+1}$. By the colon capturing property, $z \in$ $\left(x_{1}, \ldots, x_{i}\right)^{*}$. But for parameter ideals, tight closure is the same as the contraction of the expansion to $R^{+}$(see the discussion of Property 2 ). Thus $z \in\left(x_{1}, \ldots, x_{i}\right) R^{+} \cap R$. This holds for all $i$, so $x_{1}, \ldots, x_{d}$ is a regular sequence on $R^{+}$, and the Theorem is "proved". Unfortunately, this is not an honest proof because the proof that $I^{*}=I R^{+} \cap R$ for parameter ideals $I$ uses the Cohen-Macaulayness of $R^{+}$.

\section{Property 4: Relationship to integral closure.}

Property 4 is really two statements. First, the tight closure is contained in the integral closure for any ideal $I$. Second, the integral closure of $I^{\mu}$ (where $\mu$ is the least number of generators of $I$ ) is contained in the tight closure $I^{*}$.

The point in proving both statements is the following alternative definition of the integral closure $\bar{J}$ of an ideal $J$ in a domain $R$ : an element $z \in \bar{J}$ if and only if there exists a non-zero $c$ in $R$ such that $c z^{n} \in J^{n}$ for all (equivalently, for infinitely many) $n \gg 0$. (This can be easily proved equivalent to the more standard definition of integral closure by recalling another characterization of integral closure: $\bar{J}$ consists of all elements $z$ such that $z \in J V$ for all discrete valuation rings $V$ lying between $R$ and its fraction field. ) Note that in particular, $I^{*}=\bar{I}$ for all principal ideals $I$.

Now, with this definition of the integral closure, it is immediately clear that the tight closure of any ideal is contained in the integral closure. Indeed, since the $p^{e}-t h$ power of the generators of $I$ are contained in the $p^{e}-t h$ power of $I$, we have

$$
c z^{p^{e}} \in I^{\left[p^{e}\right]} \subset I^{p^{e}}
$$


for all $e$. So any $z$ in $I^{*}$ is in $\bar{I}$.

For the second statement, suppose that $z \in \overline{I^{\mu}}$. This means that there exists a non-zero $c$ such that for all $n, c z^{n} \in I^{\mu n}$. If $y_{1}, \ldots, y_{\mu}$ generate $I$, then $I^{\mu n}$ is generated by monomials of degree $\mu n$ in the $y_{i}$. But if $y_{1}^{a_{1}} y_{2}^{a_{2}} \ldots y_{\mu}^{a_{\mu}}$ is such a monomial, at least one $a_{i}$ must be greater than or equal to $n$. So

$$
c z^{n} \in I^{\mu n} \subset\left(y_{1}^{n}, \ldots, y_{\mu}^{n}\right)
$$

for all $n$. In particular, this holds for $n=p^{e}$, for all $e$, and we conclude that $z \in I^{*}$. The proof that $\overline{I^{\mu}} \subset I^{*}$ is complete.

The statement that $\overline{I^{\mu}} \subset I^{*}$ is sometimes called the Briançon-Skoda Theorem. The original Briançon-Skoda Theorem stated that for any ideal $I$ in a ring of convergent complex power series, the integral closure of the $\mu$-th power of $I$ is contained in $I$, where $\mu$ is the minimal number of generators of $I$ [ 何. This statement was later generalized by Lipman and Sathaye to more general regular local rings and then by Lipman and Tessier to certain ideals in the the so-called 'pseudo-rational' local rings (for a ring essentially of finite type over a field of characteristic zero, pseudo-rational is equivalent to rational singularities) [30], 331. Tight closure gives an extremely simple proof of the Briançon-Skoda theorem for any regular ring containing a field: $\overline{I^{\mu}} \subset I^{*} \subset I$, where the first inclusion follows from Property 4 and the second by Property 1. But better still, tight closure explains what happens in a non-regular ring as well.

The original motivating problem for the Briançon-Skoda theorem is said to be due to J. Mather: if $f$ is a germ of an analytic function vanishing at the origin in $\mathbb{C}^{n}$, find a uniform $k$ (depending only on $n$ ) such that $f^{k}$ is in the ideal generated by the partial derivatives of $f$. The Briançon-Skoda theorem tell us that we can take $k=n$. Indeed, it is easy to check that $f \in \overline{J_{f}}=\overline{\left(\frac{\partial f}{\partial x_{1}}, \ldots, \frac{\partial f}{\partial x_{n}}\right)}$. So $f^{n} \in{\overline{J_{f}}}^{n} \subset \overline{J_{f}^{n}} \subset J_{f}$. It is remarkable how easy the tight closure proof is for this problem that once seemed very difficult.

Before moving on to Property 5, we consider one more comparison of tight and integral closure. Let $I$ be an $m$-primary ideal in a local domain of dimension $d$. Recall the Hilbert-Samuel function defined by

$$
H S(n)=\operatorname{length} R / I^{n} .
$$

This function is eventually a polynomial in $n$, and its normalized leading coefficient

$$
\lim _{n \rightarrow \infty} \frac{d !}{n^{d}} H S(n)
$$

is called the Hilbert-Samuel multiplicity of $I$. Analogously, when $R$ is of characteristic $p$, we can define the Hilbert-Kunz function

$$
H K(e)=\operatorname{length} R / I^{\left[p^{e}\right]} .
$$

This function has polynomial growth in $p^{e}$, and its leading coefficient

$$
\lim _{n \rightarrow \infty} \frac{1}{\left(p^{e}\right)^{d}} H K(e)
$$


is called the Hilbert-Kunz multiplicity of $I$.

As is well known, the integral closure of $I$ is the largest ideal containing $I$ having the same Hilbert-Samuel multiplicity (assuming the completion of $R$ is equidimensional). What is also fairly straightforward to prove is that the tight closure of $I$ is the largest ideal containing $I$ having the same Hilbert-Kunz multiplicity (assuming the completion of $R$ is reduced and equidimensional) [18]. In this sense, tight closure is a natural analog of integral closure.

Hilbert-Kunz functions are interesting and mysterious, with important connections to tight closure theory and surprising interactions with number theory. Much has been proved about them by Paul Monsky, among others; see, for example, 36]. and to the bibliography of [23] for more references on this topic.

\section{Property Five: Persistence of Tight Closure.}

The persistence property states: whenever $R \rightarrow S$ is a map of rings containing a field, $I^{*} S \subset(I S)^{*}$. In other words, any element in the tight closure of an ideal $I$ of $R$ will "persist" in being in the tight closure of $I$ after expansion to any $R$-algebra.

Before discussing the precise hypothesis necessary, let us consider what is involved in proving such a statement. Suppose $z \in I^{*}$ where $I$ is an ideal in domain $R$. Thus there exists a non-zero $c$ such that

$$
c z^{\left[p^{e}\right]} \in I^{\left[p^{e}\right]}
$$

for all large $e$. Expanding to $S$, of course, the same relationship holds in $S$ (using the same letters to denote the images of $c, z$, and $I$ in $S$ ). This would seem to say that the image of $z$ is in $(I S)^{*}$, which is what we need to show. The problem is that $c$ may be in the kernel of the map $R \rightarrow S$. Thus we need to find a $c$ that "witnesses" $z \in I^{*}$ but is not in this kernel.

Unlike the first four properties, Property 5 does not follow immediately from the definition. The new idea we need is the idea of a test element.

DEFINITION 2.7 An element $c$ in a prime characteristic ring $R$ is said to be in the test ideal of $R$ if, for all ideals $I$ and all elements $z \in I^{*}$, we have $c z^{p^{e}} \in I^{\left[p^{e}\right]}$ for all $e$. An element $c$ is a test element if it is in the test ideal but not in any minimal prime of $R$.

Note that the definition of the test ideal requires that $c z^{p^{e}} \in I^{\left[p^{e}\right]}$ for all $e$, not just for all sufficiently large $e$. We could also define the asymptotic test ideal as above but require only that $c z^{p^{e}} \in I^{\left[p^{e}\right]}$ for $e \gg 0$. An interesting fact is that the aymptotic test ideal is a $D$-module - that is, it is a submodule of the module $R$ under the action of the ring of all $\mathbb{Z}$-linear differential operators on $R$. See [39].

It is not at all obvious that there exists a non-zero test ideal for a ring $R$. Fortunately, however, it is not very difficult to prove the following.

THEOREM 2.8 [19 Let $R$ be a ring of prime characteristic, and assume that the Frobenius map of $R$ is finite. If $c$ is an element of $R$ such that the localization $R_{c}$ is regular, then $c$ has a power which is a test element. That is, the test ideal contains an ideal defining the non-regular locus of Spec $R$. 
In a later paper, Hochster and Huneke prove this without the assumption that the Frobenius map is finite, imposing the weaker and more technical hypothesis of being finitely generated over an excellent local ring. Although the theorem stated above for rings in which Frobenius is finite is quite easy to prove, the proof in the more general setting is difficult and technical; see 21].

Note that in any ring $R$, the element 1 is a test element if and only if $R$ is weakly F-regular. We expect that much more is true:

Conjecture. The test ideal defines precisely the non-F-regular locus in Spec $R$.

The conjecture is proved in some cases, such as for (excellent local) Gorenstein rings [21] and for rings $\mathbb{N}$-graded over a field [32].

Having introduced the idea of a test element, we resume our discussion of persistence. First of all, we should say that Property 5 is not known to hold in the generality we've stated; some mild hypothesis on $R$ is needed. The problem is in finding test elements for $R$.

Let us now sketch the proof of persistence. Let $R \stackrel{\phi}{\rightarrow} S$ be a map of domains. $\stackrel{2}{I}^{2}$ As we remarked above, persistence is trivially true when $\phi$ is injective, so factoring $\phi$ as a surjection followed by an injection, we might as well assume $\phi$ is surjective. Now factor $\phi$ as a sequence of surjections

$$
R \rightarrow R / P_{1} \rightarrow R / P_{2} \rightarrow \cdots \rightarrow R /(\operatorname{ker} \phi)=S,
$$

where $P_{1} \subset P_{2} \subset \cdots \subset(\operatorname{ker} \phi)$ is a saturated chain of prime ideals contained in the kernel of $\phi$. By considering each map separately, we see that we might as well assume that the kernel of the map $R \stackrel{\phi}{\rightarrow} S$ has height one.

Now if $R$ is normal, then the non-regular locus of $R$ is defined by an ideal $J$ of height two or more. But as we mentioned above, this means that the test ideal has height two or more, ${ }^{3}$ so we can find a $c$ which is a test element but not in the kernel of $\phi$. The proof is complete in the case $R$ is normal.

Finally, it is not difficult to reduce the problem to the case where $R$ is normal, using Property 2. What happens is the normalization $\tilde{R}$ of $R$ maps to an integral extension $\tilde{S}$ of $S$, namely the domain $\tilde{S}$ obtained by killing a prime of $\tilde{R}$ lying over the kernel of $\phi$. The map $\tilde{R} \stackrel{\tilde{\phi}}{\rightarrow} \tilde{S}$ restricts to the map $R \stackrel{\phi}{\rightarrow} S$. Now if $z \in I^{*}$ in $R$, then $z \in(I \tilde{R})^{*}$, and so $\tilde{\phi}(z) \in(I \tilde{S})^{*}$ because we know persistence holds when the source ring is normal. By Property 2 (or really, by the same proof used to prove Property 2), we see that $\phi(z) \in(I \tilde{S})^{*} \cap S \subset(I S)^{*}$. This completes the proof of persistence.

We have completed the proofs and discussion of the five main properties of tight closure. It is natural to ask whether the five main properties characterize tight closure. They do not, or at least, not obviously. For example, in characteristic $p$, the 'plus closure' $I R^{+} \cap R$ satisfies Properties 1,2,3, and 5, and in all cases where it

\footnotetext{
2 Like most proofs in tight closure theory, the proof reduces immediately to the case where both $R$ and $S$ are domains.

${ }^{3}$ This requires some hypothesis on $R$, such as finite generation over an excellent local ring, so that $R$ satifies the conclusion of Hochster and Huneke's theorem about test elements above. In practice, all rings we run across will satisfy this hypothesis.
} 
can be checked, it satisfies Property 4 as well. On the other hand, since we expect $I^{*}=I R^{+} \cap R$, this is perhaps not very convincing.

A more interesting question is whether we can define a closure operation for rings that do not contain a field (that is, in 'mixed characteristic') which satisfies Properties 1 through 5 . If so, many theorems that can now be proved only for rings containing a field, such as the homological conjectures that grew out of Serre's work on multiplicities, would suddenly admit "tight closure" proofs. The only serious attempts at defining such a closure operation in mixed characteristic are due to Mel Hochster, but so far none has proved successful; see, for example, [16.

I hope it is clear from section one that the main ideas in tight closure theory are remarkably simple and elegant, and also that they have far-reaching consequences. In section two, we will look more closely at applications of tight closure to algebraic geometry.

\section{THREE APPLICATIONS OF TIGHT CLOSURE}

At the beginning of the part one, we mentioned that tight closure is applicable to a wide range of problems in commutative algebra and related fields. Now we will discuss in greater detail how tight closure has increased our insight in three areas of algebraic geometry: adjoint linear systems (Fujita's Freeness Conjecture), vanishing theorems for cohomology (Kodaira Vanishing), and singularities. We will mainly discuss the first of these, giving a tight closure proof of Fujita's freeness conjecture for globally generated line bundles, but we point out connections with the other two topics as they arise.

In all three areas, characteristic $p$ methods are used to prove characteristic zero theorems. The unifying theme for the tight closure approach to these three problems is the action of the Frobenius operator on local cohomology.

\section{Reduction to Characteristic $p$.}

Reduction to characteristic $p$ is easiest to understand by example. Say we want to study the affine scheme associated to the ring

$$
\frac{\mathbb{Q}[x, y, z]}{\left(x^{3}+y^{3}+z^{3}\right)} \text {. }
$$

We instead consider the "fibration"

$$
\operatorname{Spec} \frac{\mathbb{Z}[x, y, z]}{\left(x^{3}+y^{3}+z^{3}\right)} \rightarrow \text { Spec } \mathbb{Z} \text {. }
$$

The fiber over a closed point $(p) \in \operatorname{Spec} \mathbb{Z}$ is the characteristic $p$ scheme

$$
\operatorname{Spec} \frac{\mathbb{Z} /(p)[x, y, z]}{\left(x^{3}+y^{3}+z^{3}\right)},
$$

whereas the fiber over the generic point $(0) \in \operatorname{Spec} \mathbb{Z}$ is the original scheme

$$
\operatorname{Spec} \frac{\mathbb{Q}[x, y, z]}{\left(x^{3}+y^{3}+z^{3}\right)} .
$$


For the sorts of questions we are interested in here (which are ultimately cohomological) the following philosophy holds: what is true for the generic fiber is true for a Zariski dense set of closed fibers, and conversely, what is true for a Zariski dense set of closed fibers is true for the generic fiber. So in order to study the ring $\frac{\mathbb{Q}[x, y, z]}{\left(x^{3}+y^{3}+z^{3}\right)}$, we consider the ring $\frac{\mathbb{Z} /(p)[x, y, z]}{\left(x^{3}+y^{3}+z^{3}\right)}$ for a "generic" $p$.

The same approach works even if we take $\mathbb{C}$ as the ground field. Indeed,

$$
\text { Spec } \frac{\mathbb{C}[x, y, z]}{\left(x^{3}+y^{3}+z^{3}\right)},
$$

is obtained from Spec $\frac{\mathbb{Q}[x, y, z]}{\left(x^{3}+y^{3}+z^{3}\right)}$ by the flat base change $\mathbb{Q} \rightarrow \mathbb{C}$. Again, from the point of view of the types of questions we will consider, we might as well study $\frac{\mathbb{Q}[x, y, z]}{\left(x^{3}+y^{3}+z^{3}\right)}$, and hence $\frac{\mathbb{Z} /(p)[x, y, z]}{\left(x^{3}+y^{3}+z^{3}\right)}$ for a "generic" $p$.

The philosophy holds for any scheme of finite type over a field of characteristic zero. For example, if we are interested in the ring

$$
R=\frac{\mathbb{C}[x, y, z]}{\left(\pi x^{3}+\sqrt{17} y^{3}+z^{3}\right)},
$$

we set $A=\mathbb{Z}[\pi, \sqrt{17}]$ and consider the fibration

$$
\operatorname{Spec} \frac{A[x, y, z]}{\left(\pi x^{3}+\sqrt{17} y^{3}+z^{3}\right)} \rightarrow \operatorname{Spec} A .
$$

Again, we might as well study the prime characteristic ring $\frac{A / \mu[x, y, z]}{\left(\pi x^{3}+\sqrt{17} y^{3}+z^{3}\right)}$, where $\mu$ is a generic maximal ideal in $A$. Each $A / \mu$ is a finite field, so these closed fibers are all "characteristic $p$ models", for varying $p$, of the original ring $R$.

In general, if

$$
R=\frac{k\left[x_{1}, \ldots, x_{n}\right]}{\left(F_{1}, \ldots, F_{r}\right)}
$$

where $k$ is a field of characteristic zero, we let $A=\mathbb{Z}\left[\right.$ coefficients of the $F_{i}$ 's] $\subset k$ and set

$$
R_{A}=\frac{A\left[x_{1}, \ldots, x_{n}\right]}{\left(F_{1}, \ldots, F_{r}\right)}
$$

Then the map

$$
\text { Spec } R_{A} \rightarrow \operatorname{Spec} A
$$

(or the map $A \rightarrow R_{A}$ ) will be called a family of models for Spec $R$ (or $R$ ). The generic fiber is the original scheme Spec $R$ (after extending the field if necessary) and a generic (or typical) closed fiber is a characteristic $p$ model of Spec $R$. We will prove theorems about $R$ by establishing the same statement for a generic characteristic $p$ model of $R$, that is, "for all large $p . "$

The idea of a family of models can be used to define concepts in characteristic zero which seemingly only make sense in prime characteristic. For example, we can define F-regularity and F-rationality for finitely generated algebras over a field in this way. 
DEFINITION 3.1 Let $R$ be a finitely generated algebra over a field of characteristic zero. Then $R$ is said to have F-regular type if $R$ admits a family of models $A \rightarrow R_{A}$ in which a Zariski dense set of closed fibers are F-regular. (This does not depend on the choice of the the family of models.)

Similarly, we can define weakly F-regular type, F-rational type, or F-split type for any finitely generated algebra over a field of characteristic zero. (In characteristic $p, \mathrm{~F}$-split means that the Frobenius map splits, that is, $R^{p} \subset R$ splits as a $R^{p}$-module map.)

There is a subtlety in the meaning of F-regularity for algebras of characteristic zero. As we've said in part one, the operation of tight closure can be defined for any ring containing a field, so it makes sense to define a finitely generated algebra over a field of characteristic zero to be weakly F-regular if all ideals are tightly closed. This is a priori different from the condition of weakly F-regular type. We expect that these notions are equivalent, but this remains unsolved. See [17].

The notions of F-rational type and F-regular type turn out to be intimately connected with the singularities that come up in the minimal model program. The first theorem in this direction explains the name "F-rational".

THEOREM 3.2 [40, 11] A finitely generated algebra over a field of characteristic zero has F-rational type if and only if it has rational singularities.

The concept of rational singularities is very important in birational algebraic geometry. Recall that by definition, a ring $R$ has rational singularities if and only if it is normal and it admits a desingularization $X$ for which $H^{i}\left(X, \mathcal{O}_{X}\right)=0$ for all $i>0$.)

We will not dwell on this theorem here, rather refering the the papers 40 and [11] in the bibliography. Later, we will later mention some ideas in the proof. Now we move on the application of tight closure to Fujita's freeness conjecture, where many related ideas appear.

\section{Application of Tight Closure to Adjoint Linear Series.}

Let $X$ be a smooth projective variety of dimension $d$, and let $\mathcal{L}$ be an ample invertible sheaf on $X$. We are interested in the adjoint line bundles $\omega_{X} \otimes \mathcal{L}^{n}$, for $n>0$. Because $\mathcal{L}$ is ample, we know that for large $n$, this adjoint bundle is globally generated. Fujita's freeness conjecture provides an effective version of this statement.

Fujita's Freeness Conjecture. With $X$ and $\mathcal{L}$ as above, the sheaf $\omega_{X} \otimes \mathcal{L}^{d+1}$ is globally generated.

The conjecture is known in characteristic zero in dimension up to four [37, [7], [26]. See [28] for a survey. In arbitrary characteristic, the best that is known is given by the following theorem.

THEOREM 3.3 [41 If $X$ is a smooth projective variety of dimension $d$ and $\mathcal{L}$ is a globally generated ample line bundle on $X$, then $\omega_{X} \otimes \mathcal{L}^{d+1}$ is globally generated. 
See [4] for a recent improvement of this result.

Our next task is to prove this theorem, that is, to establish Fujita's Freeness Conjecture for globally generated line bundles. This will give a good overview of some of the methods that can be used in applying tight closure to algebro-geometric questions.

If $X$ has characteristic zero, the first step is to reduce to the characteristic $p$ case using the standard technique we described. So it is enough to prove the theorem in the case that $X$ has prime characteristic.

A good way to study an ample line bundle on a projective variety $X$ is to build the section ring

$$
S=\oplus_{n \geq 0} H^{0}\left(X, \mathcal{L}^{n}\right) .
$$

This is a finitely generated, $\mathbb{N}$-graded ring whose associated projective scheme recovers $X$. Its dimension is $d+1$. Assuming that $X$ is irreducible, every section ring $S$ will be a domain. If $X$ is smooth, then $S$ has (at worst) an isolated singularity at the unique homogeneous maximal ideal $m$. The invertible sheaf $\mathcal{L}^{n}$ corresponds to the graded $S$-module $S(n)$, the $S$-module $S$ with degrees shifted by n.

Fujita's Freeness Conjecture is equivalent to the following more commutative algebraic statement.

Fujita's Freeness Conjecture in terms of local cohomology If $(S, m)$ is a section ring with an isolated non-smooth point, then $H_{m}^{d+1}(S)$ has the following property: there exists an integer $N$ such that for all $\eta \in H_{m}^{d+1}(S)$ of degree less than $N, \eta$ has a non-zero $S$-multiple of degree $-d-1$.

The proof of the equivalence of this statement with Fujita's Conjecture is not difficult. This is essentially the dual statement (using Matlis duality for $S$ or Serre duality for $X$ ). Details can be found in [11.

To prove Fujita's Conjecture, we will tackle this local cohomological conjecture. First we describe a convenient way to think about elements in the local cohomology module $H_{m}^{d+1}(S)$.

Let $x_{0}, x_{1}, \ldots, x_{d}$ be a system of parameters for $S$ of degree one. Such a system of parameters exists by our assumption that $\mathcal{L}$ is globally generated (after enlarging the ground field if necessary). The local cohomology module $H_{m}^{d+1}(S)$ can be computed as the cokernel of the following map

$$
\begin{aligned}
S_{x / x_{0}} \oplus S_{x / x_{1}} \oplus \cdots \oplus S_{x / x_{n}} & \longrightarrow S_{x} \\
\left(\frac{s_{0} x_{0}^{t}}{x^{t}}, \frac{s_{1} x_{1}^{t}}{x^{t}}, \ldots, \frac{s_{d} x_{d}^{t}}{x^{t}}\right) & \mapsto \frac{\sum_{i=0}^{d}(-1)^{i} s_{i} x_{i}^{t}}{x^{t}}
\end{aligned}
$$

where $x$ denotes the product $x_{0} x_{1} \ldots x_{d}$ of the $x_{i}$ 's. This is the last map in the Cech complex for computing the cohomology of the sheaf of $\mathcal{O}_{X}$-algebras $\bigoplus_{n=0}^{\infty} \mathcal{O}_{X}(n L)$ with respect to the affine cover of $X$ given by the $d+1$ open sets $U_{i}$ where $x_{i}$ does not vanish. More generally, the local cohomology modules $H_{m}^{i}(S)$ can be computed as the cohomology of this Cech complex, so that $H_{m}^{i}(S)=\bigoplus_{n \in \mathbb{Z}} H^{i-1}\left(X, \mathcal{L}^{n}\right)$, for all $i>1$. 
We represent elements of $H_{m}^{d+1}(S)$ by fractions $\left[\frac{z}{x^{t}}\right]$, with the square bracket reminding us of the equivalence relation on fractions. If the degree of $\eta$ is $-n$, we see that $-n=\operatorname{deg} z-t(d+1)$.

It is easy to see that if $z \in\left(x_{0}^{t}, x_{1}^{t}, \ldots, x_{d}^{t}\right)$, then $\eta=\left[\frac{z}{x^{t}}\right]$ must be zero, by thinking about the image of the map above. Unfortunately, the converse is false. However, we have the following interesting observation.

LEMMA 3.4 If $\eta=\left[\frac{z}{x^{t}}\right]=0$, then $z \in\left(x_{0}^{t}, \ldots, x_{d}^{t}\right)^{*}$.

The Lemma is easily proved: if $\eta=\left[\frac{z}{x^{t}}\right]=0$, then this means that for some integer $s$, we have

$$
\left[\frac{z}{x^{t}}\right]=\left[\frac{x^{s} z}{x^{t+s}}\right]=0
$$

where now $x^{s} z \in\left(x_{0}^{t+s}, \ldots, x_{d}^{t+s}\right)$. Thus

$$
z \in\left(x_{0}^{t+s}, \ldots, x_{d}^{t+s}\right): x^{s}
$$

so by colon capturing, $z \in\left(x_{0}^{t}, \ldots, x_{d}^{t}\right)^{*}$. The Lemma is proved.

\section{The Frobenius Action on Local Cohomology.}

The Frobenius action on local cohomology is the main idea in the proof of Fujita's Freeness Conjecture for globally generated line bundles, and in the proof of the equivalence of rational singularities with F-rationality. It is also the central point in the relationship between tight closure and the Kodaira Vanishing theorem. The idea of using the Frobenius action on local cohomology to study tight closure first appears in the work of Richard Fedder and Kei-ichi Watanabe [9].

The Frobenius action

$$
H_{m}^{d+1}(S) \stackrel{F}{\rightarrow} H_{m}^{d+1}(S)
$$

is easy to understand. Indeed, Frobenius acts in a natural way on each module $S_{x_{i_{1}} \ldots x_{i_{r}}}$ in the Cech complex defining the local cohomology modules; it simply raises fractions to their $p-t h$ powers. This action obviously commutes with the boundary maps, so that it induces a natural action on the local cohomology modules. In particular, the Frobenius action on $H_{m}^{d+1}(S)$ is given by

$$
\eta=\left[\frac{z}{x^{t}}\right] \mapsto \eta^{p}=\left[\frac{z^{p}}{\left(x^{t}\right)^{p}}\right] .
$$

Using this, it makes sense to define tight closure for submodules of $H_{m}^{d+1}(S)$ by mimicking the definition for ideals. For example, we can define the tight closure of the zero submodule in $H_{m}^{d+1}(S)$ :

$$
0^{*}=\left\{\eta \in H_{m}^{d+1}(S) \mid \text { there exists } c \neq 0 \text { with } c \eta^{p^{e}}=0 \text { for all } e \gg 0\right\} .
$$

The tight closure of zero in $H_{m}^{d+1}(S)$ is an important gadget. One can show that it is the unique maximal proper submodule of $H_{m}^{d+1}(S)$ stable under the action of Frobenius [40].

Returning to the proof of Fujita's Freeness Conjecture, we observe the following two facts. 
(1) $\eta=\left[\frac{z}{x^{t}}\right] \in 0^{*}$ if and only if $z \in\left(x_{0}^{t}, \ldots, x_{d}^{t}\right)^{*}$.

(2) Any test element $c$ kills $0^{*}$.

These two facts are straightforward to prove using nearly the same argument as in the proof of the Lemma above.

Now the proof can be summarized in two main steps. First we show that if $\eta \in H_{m}^{d+1}(S)$ does not have a multiple of degree $-d-1$, then $\eta$ is in $0^{*}$. Next we show that $0^{*}$ vanishes in all sufficiently small degrees. Obviously, upon completion of these two steps, the proof is complete.

Step One: if $\eta \in H_{m}^{d+1}(S)$ does not have a multiple of degree $-d-1$, then $\eta$ is in $0^{*}$.

The main point is colon capturing. Assume on the contrary, that an element $\eta=\left[\frac{z}{x^{t}}\right]$ of degree $-n$ has no non-zero multiple of degree $-d-1$. This means that every element of $S$ of degree $n-d-1$ must kill $\eta$. In particular,

$$
\left(x_{0}, \ldots, x_{d}\right)^{n-d-1} \eta=0 .
$$

By the Lemma, this means that

$$
\left(x_{0}, \ldots, x_{d}\right)^{n-d-1} z \in\left(x_{0}^{t}, \ldots, x_{d}^{t}\right)^{*},
$$

or in other words,

$$
z \in\left(x_{0}^{t}, \ldots, x_{d}^{t}\right)^{*}:\left(x_{0}, \ldots, x_{d}\right)^{n-d-1} .
$$

Now we use colon capturing. We manipulate the parameters $x_{0}, \ldots, x_{d}$ formally as if they are the indeterminants of a polynomial ring, in which case the colon ideal (ignoring the $*$ ) would be easily computed to be

$$
\left(x_{0}^{t}, \ldots, x_{d}^{t}\right)+\left(x_{0}, \ldots, x_{d}\right)^{(d+1)(t-1)-(n-d-1)+1} .
$$

Colon capturing says that the actual colon ideal is contained in the tight closure of this "formal" colon ideal, that is,

$$
z \in\left[\left(x_{0}^{t}, \ldots, x_{d}^{t}\right)+\left(x_{0}, \ldots, x_{d}\right)^{(d+1)(t)-(n)+1}\right]^{*} .
$$

But note that the degree $z$ is $(d+1) t-n$ (because $\eta=\left[\frac{z}{x^{t}}\right]$ has degree $-n=$ $\operatorname{deg} z-(d+1) t)$. Thus

$$
z \in\left[\left(x_{0}^{t}, \ldots, x_{d}^{t}\right)+\left(x_{0}, \ldots, x_{d}\right)^{\operatorname{deg} z+1}\right]^{*} .
$$

A moment's thought reveals that this forces

$$
z \in\left(x_{0}^{t}, \ldots, x_{d}^{t}\right)^{*} .
$$

Indeed, if

$$
c z^{q} \in\left(x_{0}^{t}, \ldots, x_{d}^{t}\right)^{[q]}+\left[\left(x_{0}, \ldots, x_{d}\right)^{\operatorname{deg} z+1}\right]^{[q]},
$$

we see immediately that because the degree of $c$ is fixed, the degrees of the generators of $\left[\left(x_{0}, \ldots, x_{d}\right)^{\operatorname{deg} z+1}\right]^{[q]}$ are much larger than the degree of $c z^{q}$, so that $c z^{q}$ must 
in fact be in the ideal $\left(x_{0}^{t}, \ldots, x_{d}^{t}\right)^{[q]}$ for large $q=p^{e}$. But by Fact (1) above, then we see that

$$
\eta=\left[\frac{z}{x^{t}}\right] \in 0^{*},
$$

and the proof of step one is complete.

Step two: $0^{*}$ vanishes in sufficiently small degrees.

The point is to consider the test elements of $S$. Because $X$ is smooth, the section ring $S$ has an isolated singularity. This means that the defining ideal of the nonregular locus of $S$ is $m$-primary. As we mentioned in Lecture 1, this implies that the test ideal of $S$ (of all elements that "witness" all tight closure relations) contains an $m$-primary ideal. But according to Fact 2 above, the test ideal of $S$ annihilates $0^{*}$, so that $0^{*}$ is killed by an $m$-primary ideal. This says that $0^{*}$ has finite length, so of course, it must vanish eventually in all degrees sufficiently small. This completes the proof of step two, and thus the proof of Fujita's Freeness Conjecture for globally generated line bundles.

Experts will notice that the argument above does not really require that $X$ be smooth. We used smoothness only in Step 2, to conclude that $0^{*}$ is finite length. But $0^{*}$ is of finite length more generally, and is in fact equivalent to the variety $X$ being F-rational (or F-rational type in characteristic zero). Thus Fujita's Freeness Conjecture holds for any globally generated ample line bundle on a projective Frational (type) variety.

We should remark that Fujita's Freeness Conjecture for globally generated line bundles can also be proved, in characteristic zero, using the Kodaira vanishing theorem. As far as I know, however, tight closure provides the only proof in prime characteristic. Interestingly, the Frobenius action on local cohomology seems to act as a substitute for Kodaira Vanishing. There is a good reason for this: it turns out that Kodaira vanishing theorem is equivalent to a statement about the action on Frobenius on local cohomology modules.

\section{Tight Closure and Kodaira Vanishing.}

Recall the classical Kodaira Vanishing Theorem:

Kodaira Vanishing If $X$ is a smooth projective variety of characteristic zero, and $\mathcal{L}$ is any ample invertible sheaf on $X$, then $H^{i}\left(X, \mathcal{L}^{-1}\right)=0$ for all $i<\operatorname{dim} X$.

The Kodaira Vanishing Theorem is false in characteristic $p$, although it can be proved by reduction to characteristic $p$ [6]. See also [8].

Let $S=\oplus_{n \geq 0} H^{0}\left(X, \mathcal{L}^{n}\right)$ be the section ring of the pair $(X, \mathcal{L})$. Unwinding definitions using the point of view that local cohomology can be computed from the Cech complex of the $\mathcal{O}_{X}$-algebra $\oplus \mathcal{L}^{n}$, Kodaira Vanishing is seen to be equivalent to

$$
H_{m}^{i}(S) \text { vanishes in negative degree for all } i \text { with } 1<i<\operatorname{dim} S .
$$

Because $S$ has (at worst) an isolated non-Cohen-Macaulay point at $m$, we know that each $H_{m}^{i}(S)$ is supported at $m$, and hence must vanish in degrees sufficiently small. So we could state the Kodaira Vanishing Theorem as follows: the Frobenius 
action on a dense set of characteristic $p$ models for $S$ is injective in negative degrees on $H_{m}^{i}(S)$, for $1<i<\operatorname{dim} S$.

Although it may sound a bit silly, this way of stating the vanishing of local cohomology in negative degree has the advantage of making sense also for the top local cohomology module $H_{m}^{\operatorname{dim} S}(S)$. In fact, the injective action of Frobenius on $H_{m}^{d+1}(S)$ in negative degrees is a new and important phenomenon, a natural generalization of the Kodaira Vanishing Theorem, which is not at all apparent otherwise. This extension to the top local cohomology module was conjectured to be true and called "Strong Kodaira Vanishing" in [2.5]. The conjecture was proved in a beautiful paper of Nobuo Hara [11], and in fact, turns out to be the main point in his proof that a rationally singular variety (of characteristic zero) must be of F-rational type. (See also [35].)

The injective action of Frobenius on the negative degree part of local cohomolgy can be re-interpreted in terms of tight closure of parameter ideals. Using ideas similar to the ideas we used in the proof of Fujita's Conjecture to translate statements about the Frobenius action on $\eta=\left[\frac{z}{x^{t}}\right]$ into statements about the tight closure of $\left(x_{0}^{t}, x_{1}^{t}, \ldots, x_{d}^{t}\right)$, we get a tight closure version of Kodaira Vanishing.

Kodaira Vanishing in terms of Tight Closure. 225] Let $S$ be a section ring of a pair $(X, \mathcal{L})$ where $X$ is a smooth variety of characteristic zero and $\mathcal{L}$ is an ample invertible sheaf of $\mathcal{O}_{X}$-modules. Then for any proper subset $x_{0}, \ldots, x_{k}$ of a system of (homogeneous) parameters for $S$, where $\operatorname{deg} x_{i} \gg 0$,

$$
\left(x_{0}, \ldots, x_{k}\right)^{*} \subset \sum_{i=0}^{k}\left(x_{0}, \ldots, \hat{x}_{i}, \ldots, x_{k}\right)^{*}+S_{\geq D}
$$

where $D$ is the sum of the degrees of the $x_{i}$ 's.

This theorem is equivalent to the Kodaira Vanishing Theorem. Just as Kodaira Vanishing can fail in prime characteristic, so can this tight closure statement. However, the statement holds when $S$ is a generic characteristic $p$ model for a section ring of characteristic zero, that is, "for large $p . "$

By allowing the possibility that we have a full system of parameters in the above version of the Kodaira Vanishing Theorem, we get the strong Kodaira Vanishing Theorem. In fact, if $x_{0}, x_{1}, \ldots, x_{d}$ is a full system of parameters for a section $S$ as above, we get a more precise statement.

Strong Kodaira Vanishing [25] [11]. Let $S$ be an $\mathbb{N}$-graded ring over a field of characteristic zero, and let $x_{0}, x_{1}, \ldots, x_{d}$ be a full system of (homogeneous) parameters for $S$, with $\operatorname{deg} x_{i} \gg 0$. Then

$$
\left(x_{0}, \ldots, x_{d}\right)^{*}=\sum_{i=0}^{d}\left(x_{0}, \ldots, \hat{x}_{i}, \ldots, x_{d}\right)^{*}+S_{\geq D}
$$

where $D$ is the sum of the degrees of the $x_{i}$ 's.

The reason we get equality here is that $S_{\geq D}$ is contained in $\left(x_{0}, \ldots, x_{d}\right)^{*}$, as can be verified with the Briançon-Skoda theorem (Property 4).

It is possible to say precisely how large the degrees of the $x_{i}$ 's must be in the statements of Kodaira and strong Kodaira vanishing in terms of tight closure. In 
both theorems, each $x_{i}$ should have degree larger than $a$, where $a$ is the a-invariant of $S$. By definition (due to Goto and Watanabe), the $a$-invariant is the largest integer $n$ such that $H_{m}^{\operatorname{dim} S}(S)$ is non-zero in degree $n$.

The strong form of Kodaira Vanishing is conjectured in [25], where the idea of the "monomial property of a $d^{+}$sequence" due to Goto and Yamagishi is used. It is proved in [25] for rings of dimension two, from which it is shown that the Kodaira Vanishing Theorem follows for any normal surface of dimension two. In full generality, however, the statement was not known until Nobuo Hara proved the injectivity of the Frobenius action on the negatively graded part of local cohomology [11]. Hara has since greatly generalized his work; see [13].

\section{Tight Closure and Singularities.}

Finally, we summarize some more connections between tight closure and singularities in algebraic geometry.

Let $X$ be a normal variety of characteristic zero. Assume that $X$ is $\mathbb{Q}$-Gorenstein, that is, that the reflexive sheaf $\omega_{X}$ represents a torsion element $K_{X}$ in the (local) class group of $X$. In other words, the Weil divisor class $K_{X}$ is assumed to have a multiple which is locally principal.

Consider a desingularization $\tilde{X} \stackrel{\pi}{\rightarrow} X$ of $X$, where the exceptional divisor is a simple normal crossings divisor with components $E_{1}, \ldots, E_{n}$. Write

$$
K_{\tilde{X}}=\pi^{*} K_{X}+\sum_{i=1}^{n} a_{i} E_{i}
$$

for some unique rational numbers $a_{i}$. To understand this expression, suppose that $r K_{X}$ is locally principal, so that it makes sense to pull it back; then compare to $r K_{\tilde{X}}$. The difference is some divisor supported on the exceptional set, hence of the form $\sum_{i=1}^{n} m_{i} E_{i}$. Dividing by $r$, we arrive at the above expression, where 'equality' means numerical equivalence of $\mathbb{Q}$-divisors. See [27].

In general, the $a_{i}$ 's can be any rational number, although if $X$ is smooth, we can easily see that each $a_{i}$ will be a positive integer. This leads us to the following restricted class of singularities.

DEFINITION 3.5 The variety $X$ has log-terminal singularities if all $a_{i}>-1$, and has log-canonical singularities if all $a_{i} \geq 1$. (This is independent of the choice of desingularization.)

The relationship to tight closure is is evidenced by the following theorem.

THEOREM 3.6 Let $X$ be a normal $\mathbb{Q}$-Gorenstein variety of characteristic zero. $X$ has F-regular type if and only if $X$ has log-terminal singularities.

This theorem follows immediately from the equivalence of rational singularities and F-rational type discussed earlier, using the "canonical cover trick". Indeed, assuming $X$ is local, set

$$
Y=\operatorname{Spec}\left\{\mathcal{O}_{X} \oplus \mathcal{O}_{X}\left(K_{X}\right) \oplus \mathcal{O}_{X}\left(2 K_{X}\right) \oplus \ldots \mathcal{O}_{X}\left((r-1) K_{X}\right)\right\}
$$


where $r$ is such that $\mathcal{O}_{X}\left(r K_{X}\right)$ is isomorphic to $\mathcal{O}_{X}$ via a fixed isomorphism (so that we can define a ring structure on $\left.\mathcal{O}_{X} \oplus \mathcal{O}_{X}\left(K_{X}\right) \oplus \mathcal{O}_{X}\left(2 K_{X}\right) \oplus \ldots \mathcal{O}_{X}\left((r-1) K_{X}\right)\right)$. The natural map $Y \rightarrow X$ is called the canonical cover of $X$. It is easy to check that when $X$ is Cohen-Macaulay, the canonical cover $Y$ is Gorenstein, and that the map is étale in codimension one. With these properties, it is not hard to show the following two facts:

(1) (Kawamata) $Y$ has rational singularities if and only if $X$ has log-terminal singularities.

(2) (K.-i. Watanabe) Y has F-rational type if and only if $X$ has F-regular type.

Thus the equivalence of F-regular type with log-terminal singularities follows from the equivalence of F-rational type with rational singularities.

There are some subtleties involved in the argument using the canonical cover. Watanabe's argument shows F-rationality for $Y$ is equivalent to strong F-regularity for $X$. Strong F-regularity is a technical condition conjectured to be equivalent to weak F-regularity (when both are defined), introduced because it, unlike weak F-regularity, is easily shown to pass to localizations 19 . However, in the case of $\mathbb{Q}-$ Gorenstein rings, weak and strong F-regularity turn out to be equivalent [33].

The first proof that F-regular type $\mathbb{Q}$-Gorenstein singularities are log-terminal is due to Kei-ichi Watanabe and uses a different argument 46]. This different argument also produces the following nice result.

THEOREM 3.7 46] Let $X$ be a variety satisfying the conditions above. If $X$ is of $F$-split type, then $X$ has log-terminal singularities. (Recall, a local ring of characteristic $p$ is F-split if the inclusion $R^{p} \hookrightarrow R$ splits as a map of $R^{p}$ modules.)

A very interesting open problem that has deep connections with number theory is the following.

Open Problem. If $X$ has log-canonical singularities, does $X$ have $F$-split type?

\section{Further Reading on Tight Closure.}

The original tight closure paper of Hochster and Huneke 18] is still an excellent introduction to the subject. There are also a number of expository articles on tight closure. Craig Huneke's book Tight Closure and its Applications [23 is an good place for a beginning commutative algebra student to learn the subject; it contains several applications more or less disjoint from the ones discussed in detail here. It also contains an appendix by Mel Hochster [17] discussing tight closure in characteristic zero. Another nice survey is 15, which contains a list of open problems; although the article is now seven years old, many of these problems remain open. A more recent view is provided by the expository article [5]. The article 442] is a survey written for algebraic geometers. Huneke's "Tight Closure and Geometry" is another nice read for algebraists 24. All these sources, but especially [23], contain long bibliographies to direct the reader to numerous research articles on tight closure. 


\section{References}

[1] I. Aberbach. Tight closure in F-rational rings. Nagoya Math. J. 135, 1994, $43-54$.

[2] I. Aberbach, M. Hochster and C. Huneke. Localization of tight closure and modules of finite phantom projective dimension. J. Reine angew., 434, 1993, $67-114$.

[3] I. Aberbach, C. Huneke and K.E. Smith. A Tight Closure Approach to Arithmetic Macaulayfication. Illinois Journal of Math, 40, 1996, 310-329.

[4] J. Briançon, H. Skoda. Sur la clôture intégrale d'un idéal de germes de fonctions holomorphes en un point de $C^{n}$. C. R. Acad. Sci. Paris Sér. A, 278, 1974, 949-951.

[5] W. Bruns. Tight Closure. Bulletin Amer. Math. Soc., 33, 1996, 447-458.

[6] P. Deligne, L. Illusie. Relèvements modulo $p^{2}$ et decomposition du complexe de de Rham. Inventiones Math., 89, 1987, 247-270.

[7] L. Ein, R. Lazarsfeld. Global generation of pluricanonical and adjoint linear series on smooth projective three-folds. Jour. of Amer. Math. Soc., 6, 1993, 875-903.

[8] H. Esnault, E. Viehweg. Lectures on vanishing theorems. Birkhauser DMV series 20, 1992.

[9] R. Fedder, K. Watanabe. A characterization of F-regularity in terms of Fpurity. in Commutative Algebra in MSRI Publications No. 15, SpringerVerlag, New York, 1989, 227-245.

[10] S. Goto, K. Yamagishi. The theory of unconditioned strong d-sequences and modules of finite local cohomology. preprint

[11] N. Hara. A Frobenius characterization of rational singularities. American Journal of Math, 1998.

[12] N. Hara. Classification of two-dimensional $F$-regular and $F$-pure singularities. Adv. Math., 133, 1998, 33-53.

[13] N. Hara. Geometric interpretation of tight closure and test modules . preprint, 1999.

[14] N. Hara. A characteristic $p$ proof of Wahl's vanishing theorem for rational surface singularities. preprint, 1999

[15] Melvin Hochster. Tight closure in equal characteristic, big Cohen-Macaulay algebras, and solid closure (in Commutative algebra: syzygies, multiplicities, and birational algebra). Contemp. Math., 159, 1994, 173-196.

[16] M. Hochster. Solid closure (in Commutative algebra: syzygies, multiplicities, and birational algebra). Contemp. Math., 159, 1994, 103-172. 
[17] M. Hochster. The notion of tight closure in equal characteristic zero. Appendix to 'Tight Closure and Its Applications', by C. Huneke, CBMS lecture notes, 88, 1996.

[18] M. Hochster, C. Huneke. Tight closure, invariant theory, and the BrianconSkoda theorem. Jour. Amer. Math. Soc., 3, 1990, 31-116.

[19] M. Hochster, C. Huneke. Tight closure and strong F-regularity. Mémoires de la Société Mathématique de France, 38, 1989, 119-133.

[20] M. Hochster, C. Huneke. Infinite integral extensions and big Cohen-Macaulay algebras. Annals of Math., 135, 1992, 53-89.

[21] M. Hochster, C. Huneke. F-regularity, test elements and smooth base change. Trans. Amer. Math. Soc., 346, 1994, 1-62.

[22] C. Huneke. Uniform bounds in Noetherian rings. Invent. Math., 107, 1992, 203-223.

[23] C. Huneke. Tight Closure and its Applications. CBMS Lecture Notes in Mathematics, 88, American Math. Soc., Providence, RI, 1996.

[24] C. Huneke. Tight Closure and Geometry. Lecture notes from Commutative algebra summer school in Barcelona, preprint.

[25] C. Huneke, K.E. Smith. Tight Closure and the Kodaira vanishing theorem. J. reine angew. Math., 484, 1997, 127-152.

[26] Y. Kawamata. On Fujita's freeness conjecture for threefolds and fourfolds. Math. Ann., 308 (3), 1997, 491-505.

[27] Y. Kawamata., K. Matsuda , K. Matsuki. Introduction to the minimal model program. Advanced Studies in Pure Math., Algebraic Geometry, Sendai 1985, 10, 1987, 283-360.

[28] Kollár. Singularities of Pairs, in Algebraic geometry — Santa Cruz 1995, 289 325, Proc. Sympos. Pure Math., 62, Part 1, Amer. Math. Soc., Providence, RI, 1997.

[29] K. Kurano. On Macaulayfication obtained by a blow-up whose center is an equi-multiple ideal, With an appendix by Kikumichi Yamagishi. J. Algebra, 190 (2), 1997, 405-434.

[30] J. Lipman, A. Sathaye. Jacobian ideals and a theorem of Briançon-Skoda. Michigan Math. J., 28, 1981, 199-222.

[31] J. Lipman, B. Teissier. Pseudo-rational local rings and a theorem of BriançonSkoda about integral closures of ideals. Michigan Math. J., 28, 1981, 97-116.

[32] G. Lyubeznik, K.E. Smith. Weak and Strong F-regularity are equivalent for graded rings. preprint.

[33] B. MacCrimmon. PhD. Thesis. University of Michigan, 1996. 
[34] H. Matsumura. Commutative Ring Theory. Cambridge University Press, Cambridge.

[35] V.B. Mehta, V. Srinivas. Asian Journal of Mathematics, 1997.

[36] P. Monsky. The Hilbert-Kunz function. Math. Ann., 263, 1983, 43-49.

[37] I. Reider. Vector bundles of rank 2 and linear systems on algebraic surfaces. Ann. of Math., 127, 1988, 309-316.

[38] K.E. Smith. Tight closure of parameter ideals. Invent. Math., 115, 1994, 4160.

[39] K.E. Smith. The D-module structure of F-split rings. Math. Research Letters, $2,1995,377-386$.

[40] K.E. Smith. F-rational rings have rational singularities. Amer. Jour. Math., 119 (1), 1997, 159-180.

[41] K.E. Smith. Fujita's conjecture in terms of local cohomology. Jour. Algebraic Geom., 6 (3), 1997, 417-429.

[42] K.E. Smith. Vanishing, singularities and effective bounds via prime characteristic local algebra, in Algebraic geometry - Santa Cruz 1995, 289-325, Proc. Sympos. Pure Math., 62, Part 1 (Please also see the erratum at http://www.math.lsa.umich.edu/ kesmith) Amer. Math. Soc., Providence, RI, 1997, 289-325.

[43] K.E. Smith. Tight closure commutes with localization in binomial rings. 1998, preprint.

[44] K.E. Smith. A tight closure proof of Fujita's Freeness Conjecture for globally generated line bundles. 1999, preprint.

[45] K.E. Smith, M. Van den Bergh. Simplicity of rings of differential operators in prime characteristic. Proc. London Math. Soc., 75 (3), 1997, 32-62.

[46] Kei-ichi Watanabe. F-purity and F-regularity vs. Log-canonical singularities, preprint. 